\title{
Effective Antenna Modellings for NF-FF Transformations with Spherical Scanning Using the Minimum Number of Data
}

\author{
Francesco D’Agostino, Flaminio Ferrara, Claudio Gennarelli, \\ Rocco Guerriero, and Massimo Migliozzi
} Department of Electronic and Computer Engineering, University of Salerno, Via Ponte Don Melillo,
I-84084 Fisciano (Salerno), Italy

Correspondence should be addressed to Claudio Gennarelli, gennar@diiie.unisa.it

Received 23 September 2010; Accepted 15 January 2011

Academic Editor: Z. Chen

Copyright () 2011 Francesco D’Agostino et al. This is an open access article distributed under the Creative Commons Attribution License, which permits unrestricted use, distribution, and reproduction in any medium, provided the original work is properly cited.

Two efficient probe-compensated near-field-far-field transformations with spherical scanning for antennas having two dimensions very different from the third one are here developed. They rely on the nonredundant sampling representations of the electromagnetic fields and on the optimal sampling interpolation expansions, and use effective antenna modellings. In particular, an antenna with a predominant dimension is no longer considered as enclosed in a sphere but in a cylinder ended in two half spheres, whereas a surface formed by two circular "bowls" with the same aperture diameter but different lateral bends is adopted to shape an antenna with two predominant dimensions. These modellings are able to fit very well a lot of antennas by properly setting their geometric parameters. It is so possible to remarkably lower the number of data to be acquired, thus significantly reducing the measurement time. Numerical tests assessing the accuracy and the robustness of the techniques are reported.

\section{Introduction}

As is well known, near-field-far-field (NF-FF) transformation techniques play a significant role in modern antenna measurements [1-4]. As a matter of fact, the pattern evaluation from NF measurements allows one to overcome those drawbacks which, for electrically large radiating systems, make it unpractical to measure the pattern in a conventional FF range. Two main techniques for the FF pattern reconstruction from NF measurements can be found in literature. The former relies on the wave-mode expansion method and uses canonical scanning surfaces (sphere, cylinder, plane) [14]. The latter is based on the equivalence theorem $[5,6]$ and can be applied to arbitrary scanning surfaces although at a remarkably increased computational cost.

Among the NF-FF transformation techniques, the employing of the spherical scanning has attracted considerable attention [7-15], since it allows one to reconstruct, from a single set of NF measurements, the complete radiation pattern of the antenna under test (AUT). In the classical NFFF transformation with spherical scanning [7-13], the choice of the highest spherical wave and the data spacings on the measurement sphere are determined according to a rule of thumb, related to the smallest sphere able to contain the AUT.

In [14], such an NF-FF transformation has been properly modified by taking into account the spatial band-limitation properties of the electromagnetic (EM) fields [16]. In particular, the choice of the highest spherical wave has been rigorously fixed by the band-limitation properties and the number of data to be acquired on the rings decreases towards the poles. Moreover, the nonredundant sampling representations of the EM field [17] have been applied in the same paper [14] to remarkably reduce the number of required NF data when antennas with one or two predominant dimensions are considered. These results have been obtained by considering the AUT as enclosed in a prolate or oblate ellipsoid, respectively. Then, an optimal sampling interpolation (OSI) formula has been developed 
to recover the data required by the aforementioned NFFF transformation from the knowledge of a nonredundant number of NF samples. However, since the case of an ideal probe has been considered in [14], the effects of the measurement probe have not been taken into account in the evaluation of the antenna FF pattern. The ideal probe assumption has been removed in [15], wherein an efficient probe-compensated NF-FF transformation technique with spherical scanning tailored for elongated or quasiplanar antennas has been developed, by properly extending the nonredundant sampling representation to the voltage measured by a nondirective probe. Then, the nonredundant voltage samples are interpolated via an efficient OSI algorithm for reconstructing the NF data required by the probe-compensated NF-FF transformation technique with spherical scanning [7], again properly modified to account for the spatial bandlimitation properties.

It must be stressed that the reduction in the number of the NF data to be acquired reflects in a decrease of the measurement time, and this is a very important issue for the antenna measurement community, since such a time is very much greater than that needed to carry out the NFFF transformation. A measurement time reduction can also be obtained by performing a fast electronic scanning via the modulated scattering technique [18], which employs arrays of scattering probes. However, antenna testing facilities based on such a technique are less flexible than those using mechanical scans. Accordingly, it appears more convenient the reduction of the needed NF data attainable by using the nonredundant sampling representations, which does not require to change the positioning systems, but only the software for controlling them.

The aim of this paper is to develop even more effective NF-FF transformations with spherical scanning tailored for antennas having one or two predominant dimensions. To this end, very flexible antenna modellings will be adopted. In particular, an electrically long antenna will be considered as enclosed in a rounded cylinder, namely, a cylinder which ends in two half spheres, whereas a surface formed by two circular "bowls" with the same aperture diameter but different lateral bends (double bowl) will be adopted to shape a quasiplanar antenna. The so-obtained NF-FF transformation techniques result to be more effective from the data reduction viewpoint than those employing the prolate or oblate ellipsoidal modelling. In fact, these flexible modellings allow one to fit the shape of a lot of actual antennas better by properly setting their geometric parameters. Moreover, they remain quite general and contain the spherical modelling as a particular case.

\section{Nonredundant Sampling Representation of the Voltage on a Sphere}

Let us consider an AUT enclosed in a convex domain bounded by a rotational surface $\Sigma$ and a nondirective probe scanning a spherical surface of radius $d$ in the antenna NF region. The spherical coordinate system $(r, \vartheta, \varphi)$ will be adopted in the following to denote an observation point $P$ in the NF region. As shown in [19], the voltage measured by such a kind of probe has the same effective spatial bandwidth of the AUT field, and, accordingly, the nonredundant sampling representations of EM fields [17] can be applied to it. Moreover, since the scanning sphere can be represented by meridians and parallels, in the following we deal with the voltage representation on an observation curve $C$ described by an analytical parameterization $\underline{r}=\underline{r}(\xi)$. According to [17], let us introduce the "reduced voltage"

$$
\tilde{V}(\xi)=V(\xi) \mathrm{e}^{j \gamma(\xi)}
$$

where $\gamma(\xi)$ is a proper phase function to be determined. The error, occurring when $\tilde{V}$ is approximated by a spatially bandlimited function, becomes negligible as the bandwidth exceeds a critical value $W_{\xi}$ [17]. In fact, it exhibits a steplike behaviour whose transition occurs at $W_{\xi}[16]$. Accordingly, such an error can be effectively controlled [20] by choosing a bandwidth equal to $\chi^{\prime} W_{\xi}$, where $\chi^{\prime}$ is an excess bandwidth factor, slightly greater than unity for electrically large antennas.

When $C$ is a meridian, by adopting

$$
W_{\xi}=\frac{\beta \ell^{\prime}}{2 \pi}
$$

( $\beta$ being the wavenumber and $\ell^{\prime}$ the length of the curve $C^{\prime}$ obtained as intersection between the meridian plane passing through the observation point $P$ and $\Sigma$ ), we get

$$
\begin{aligned}
& \gamma=\frac{\beta}{2}\left[R_{1}+R_{2}+s_{1}^{\prime}-s_{2}^{\prime}\right], \\
& \xi=\frac{\pi}{\ell^{\prime}}\left[R_{1}-R_{2}+s_{1}^{\prime}+s_{2}^{\prime}\right],
\end{aligned}
$$

where $R_{1,2}$ are the distances from $P$ to the tangency points $P_{1,2}$ on $C^{\prime}$, and $s_{1,2}^{\prime}$ are their arclength coordinates.

When the observation curve is a parallel, due to the involved symmetry, the phase function is constant and it is convenient to use the azimuthal angle $\varphi$ as parameter. The corresponding bandwidth is given [17] by

$$
\begin{aligned}
& W_{\varphi}= \frac{\beta}{2} \max _{z^{\prime}}\left(R^{+}-R^{-}\right) \\
&=\frac{\beta}{2} \max _{z^{\prime}}\left(\sqrt{\left(z-z^{\prime}\right)^{2}+\left(\rho+\rho^{\prime}\left(z^{\prime}\right)\right)^{2}}\right. \\
&\left.-\sqrt{\left(z-z^{\prime}\right)^{2}+\left(\rho-\rho^{\prime}\left(z^{\prime}\right)\right)^{2}}\right),
\end{aligned}
$$

wherein $\rho=d \sin \vartheta, \rho^{\prime}\left(z^{\prime}\right)$ is the equation of $\Sigma$ in cylindrical coordinates, and the maximum is attained [17] on that zone of the surface $\Sigma$ lying on the same side of the observation circumference with respect to its maximum transverse circle.

In the light of these results, the reduced voltage at $P(\xi, \varphi)$ on the meridian fixed by $\varphi$ can be evaluated via the OSI expansion

$$
\tilde{V}(\xi, \varphi)=\sum_{m=m_{0}-q+1}^{m_{0}+q} \tilde{V}\left(\xi_{m}, \varphi\right) \Omega_{M}\left(\xi-\xi_{m}\right) D_{M^{\prime \prime}}\left(\xi-\xi_{m}\right),
$$


where $m_{0}=\operatorname{Int}[\xi / \Delta \xi]$ is the index of sample nearest (on the left) to $P, 2 q$ is the number of the retained intermediate samples $\tilde{V}\left(\xi_{m}, \varphi\right)$, that is, the reduced voltage values at the intersection points between the sampling parallels and the considered meridian, and

$$
\begin{gathered}
\xi_{m}=m \Delta \xi=\frac{2 \pi m}{\left(2 M^{\prime \prime}+1\right)}, \\
M^{\prime \prime}=\operatorname{Int}\left(\chi M^{\prime}\right)+1, \quad M^{\prime}=\operatorname{Int}\left(\chi^{\prime} W_{\xi}\right)+1,
\end{gathered}
$$

$\chi$ being a factor that controls the truncation error [17] and $\operatorname{Int}(x)$ denoting the integer part of $x$. Moreover,

$$
\begin{gathered}
D_{M^{\prime \prime}}(\xi)=\frac{\sin \left[\left(2 M^{\prime \prime}+1\right) \xi / 2\right]}{\left(2 M^{\prime \prime}+1\right) \sin (\xi / 2)}, \\
\Omega_{M}(\xi)=\frac{T_{M}\left[2 \cos ^{2}(\xi / 2) / \cos ^{2}\left(\xi_{0} / 2\right)-1\right]}{T_{M}\left[2 / \cos ^{2}\left(\xi_{0} / 2\right)-1\right]}
\end{gathered}
$$

are the Dirichlet and Tschebyscheff Sampling functions, respectively, $T_{M}(\cdot)$ being the Tschebyscheff polynomial of degree $M=M^{\prime \prime}-M^{\prime}$ and $\xi_{0}=q \Delta \xi$.

The intermediate samples $\tilde{V}\left(\xi_{m}, \varphi\right)$ can be evaluated via the OSI formula

$$
\begin{aligned}
\tilde{V}\left(\xi_{m}, \varphi\right)= & \sum_{n=n_{0}-p+1}^{n_{0}+p} \tilde{V}\left(\xi_{m}, \varphi_{n, m}\right) \Omega_{N_{m}}\left(\varphi-\varphi_{n, m}\right) \\
& \times D_{N_{m}^{\prime \prime}}\left(\varphi-\varphi_{n, m}\right),
\end{aligned}
$$

where $\tilde{V}\left(\xi_{m}, \varphi_{n, m}\right)$ are the reduced samples on the parallel fixed by $\xi_{m}, 2 p$ is the retained samples number, $n_{0}=$ $\operatorname{Int}\left(\varphi / \Delta \varphi_{m}\right)$, and

$$
\begin{gathered}
\varphi_{n, m}=n \Delta \varphi_{m}=2 \frac{\pi n}{2 N_{m}^{\prime \prime}+1}, \quad N_{m}^{\prime \prime}=\operatorname{Int}\left(\chi N_{m}^{\prime}\right)+1, \\
N_{m}^{\prime}=\operatorname{Int}\left[\chi^{*} W_{\varphi}\left(\xi_{m}\right)\right]+1, \quad N_{m}=N_{m}^{\prime \prime}-N_{m}^{\prime}, \\
\chi^{*}=1+\left(\chi^{\prime}-1\right)\left[\sin \vartheta\left(\xi_{m}\right)\right]^{-2 / 3} .
\end{gathered}
$$

The variation of $\chi^{*}$ with $\xi$ is required to ensure a bandlimitation error constant with respect to $\xi$.

By properly matching (5) and (8), the following twodimensional OSI expansion results:

$$
\begin{aligned}
& \tilde{V}(\xi, \varphi) \\
& =\sum_{m=m_{0}-q_{+1}}^{m_{0}+q}\left\{\Omega_{M}\left(\xi-\xi_{m}\right) D_{M^{\prime \prime}}\left(\xi-\xi_{m}\right) \sum_{n=n_{0}-p+1}^{n_{0}+p}\right. \\
& \left.\quad \times \tilde{V}\left(\xi_{m}, \varphi_{n, m}\right) \Omega_{N_{m}}\left(\varphi-\varphi_{n, m}\right) D_{N_{m}^{\prime \prime}}\left(\varphi-\varphi_{n, m}\right)\right\},
\end{aligned}
$$

which makes it possible to recover the NF data needed to carry out the spherical NF-FF transformation [7] as modified in [15].

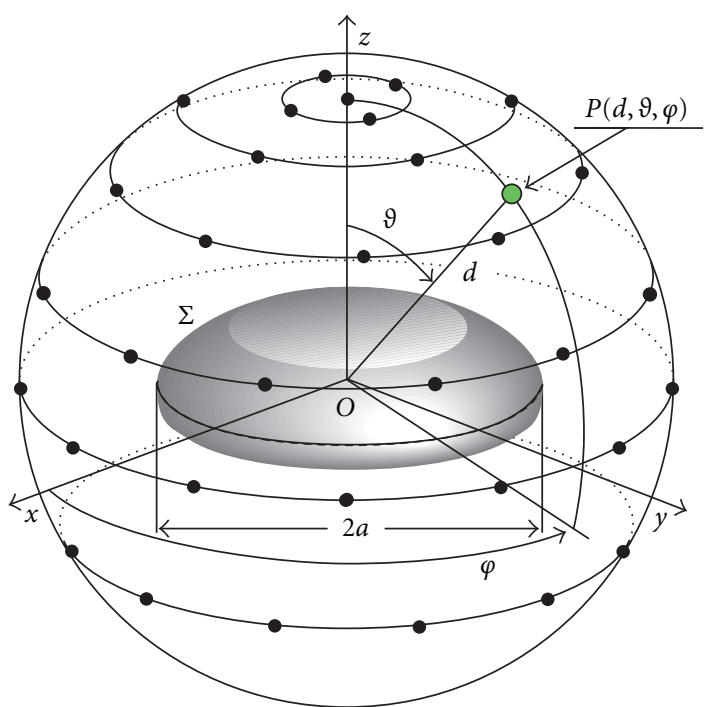

FIGURE 1: Spherical scanning for a quasiplanar antenna.

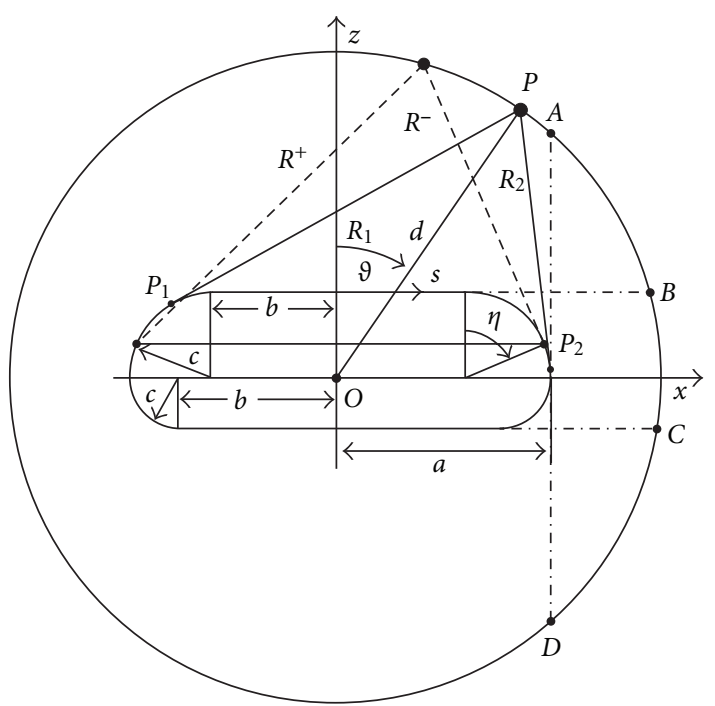

Figure 2: Relevant to the double bowl modelling.

2.1. Double Bowl Modelling. An effective and flexible modelling for an AUT having a quasiplanar geometry is obtained by choosing $\Sigma$ coincident with the smallest surface formed by two circular "bowls" with the same aperture diameter $2 a$ (see Figures 1 and 2). Note that their lateral surfaces have not the same bend because they are generally determined by rotating two different circular arcs, each equal to a quarter of circumference (with radius $c$ and $c^{\prime}$ ). This surface allows one to fit very well a lot of actual antennas by properly choosing the values of the parameters $c, c^{\prime}$, and $a$. For instance, $\Sigma$ coincides with a sphere if $c=c^{\prime}=a$, and it becomes a half sphere if $c=0$ and $c^{\prime}=a$, and it reduces to a circular dish for $c=c^{\prime}=0$. It can be easily verified that, for such a modelling, the length of the curve $C^{\prime}$ is $\ell^{\prime}=2\left[b+b^{\prime}+\left(c+c^{\prime}\right) \pi / 2\right]$, wherein $b=a-c$ and $b^{\prime}=a-c^{\prime}$. The phase function and the optimal parameter relevant to a meridian can be determined 
by substituting in relations (3) the appropriate values of $R_{1,2}$ and $s_{1,2}^{\prime}$, whose expressions change depending on the location of the tangency points $P_{1,2}$. For such a modelling, five cases must be considered for 9 ranging in $[0, \pi]$ (see Figure 2).

When $0 \leq \vartheta \leq \vartheta_{A}=\sin ^{-1}(a / d)$, it results that

$$
\begin{gathered}
R_{1}=\sqrt{d^{2}+b^{2}+2 b d \sin \vartheta-c^{2}}, \quad s_{1}^{\prime}=-\left(b+c \alpha_{1}\right), \\
\alpha_{1}=\tan ^{-1}\left(\frac{R_{1}}{c}\right)-\tan ^{-1}\left[\frac{(b+d \sin \vartheta)}{d \cos \vartheta}\right], \\
R_{2}=\sqrt{d^{2}+b^{2}-2 b d \sin \vartheta-c^{2}}, \quad s_{2}^{\prime}=b+c \alpha_{2}, \\
\alpha_{2}=\tan ^{-1}\left(\frac{R_{2}}{c}\right)-\tan ^{-1}\left[\frac{(b-d \sin \vartheta)}{d \cos \vartheta}\right] .
\end{gathered}
$$

When $\vartheta_{A}<\vartheta \leq \vartheta_{B}=\cos ^{-1}(c / d), R_{1}, s_{1}^{\prime}$ and $\alpha_{1}$ are again given by relations (11), whereas it results that

$$
\begin{gathered}
R_{2}=\sqrt{d^{2}+b^{\prime 2}-2 b^{\prime} d \sin \vartheta-c^{\prime 2}}, \quad s_{2}^{\prime}=b+c\left(\frac{\pi}{2}\right)+c^{\prime} \alpha_{2}, \\
\alpha_{2}=\tan ^{-1}\left(\frac{R_{2}}{c^{\prime}}\right)-\tan ^{-1}\left[\frac{d \cos \vartheta}{\left(d \sin \vartheta-b^{\prime}\right)}\right] .
\end{gathered}
$$

When $\vartheta_{B}<\vartheta \leq \vartheta_{c}=\pi-\cos ^{-1}\left(c^{\prime} / d\right), R_{2}, s_{2}^{\prime}$, and $\alpha_{2}$ are again given by relations (12), whereas it results that

$$
\begin{gathered}
R_{1}=\sqrt{d^{2}+b^{2}-2 b d \sin \vartheta-c^{2}}, \quad s_{1}^{\prime}=b+c\left(\alpha_{1}+\frac{\pi}{2}\right), \\
\alpha_{1}=-\tan ^{-1}\left(\frac{R_{1}}{c}\right)-\tan ^{-1}\left[\frac{d \cos \vartheta}{(d \sin \vartheta-b)}\right] .
\end{gathered}
$$

When $\vartheta_{C}<\vartheta \leq \vartheta_{D}=\pi-\sin ^{-1}(a / d), R_{1}, s_{1}^{\prime}$, and $\alpha_{1}$ are again given by relations (13), whereas it results that

$$
\begin{gathered}
R_{2}=\sqrt{d^{2}+b^{\prime 2}+2 b^{\prime} d \sin \vartheta-c^{\prime 2}}, \\
s_{2}^{\prime}=b+2 b^{\prime}+\left(c+c^{\prime}\right)\left(\frac{\pi}{2}\right)+c^{\prime} \alpha_{2}, \\
\alpha_{2}=\tan ^{-1}\left(\frac{R_{2}}{c^{\prime}}\right)-\tan ^{-1}\left[\frac{\left(d \sin \vartheta+b^{\prime}\right)}{|d \cos \vartheta|}\right] .
\end{gathered}
$$

When $\vartheta_{D}<\vartheta \leq \pi, R_{2}, s_{2}^{\prime}$, and $\alpha_{2}$ are again given by relations (14), whereas it results that

$$
\begin{gathered}
R_{1}=\sqrt{d^{2}+b^{\prime 2}-2 b^{\prime} d \sin \vartheta-c^{\prime 2}}, \\
s_{1}^{\prime}=b+c\left(\frac{\pi}{2}\right)+c^{\prime}\left(\frac{\pi}{2}-\alpha_{1}\right), \\
\alpha_{1}=\tan ^{-1}\left(\frac{R_{1}}{c^{\prime}}\right)-\tan ^{-1}\left[\frac{\left(b^{\prime}-d \sin \vartheta\right)}{|d \cos \vartheta|}\right] .
\end{gathered}
$$

For what concerns the evaluation of the maximum in (4) which allows the determination of the azimuthal bandwidth $W_{\varphi}$, it is convenient (when $\vartheta \leq \pi / 2$ ) to introduce the angular coordinate $\eta$ (Figure 2) such that $z^{\prime}=c \cos \eta$ and $\rho^{\prime}=b+c \sin \eta$. It can be easily verified through simple geometrical considerations or analytical manipulations that the sought maximum corresponds to the $\eta$ value that zeroes the derivative of $R^{+}-R^{-}$with respect to $\eta$. A quite analogous result holds when $\pi / 2<\vartheta \leq \pi$.

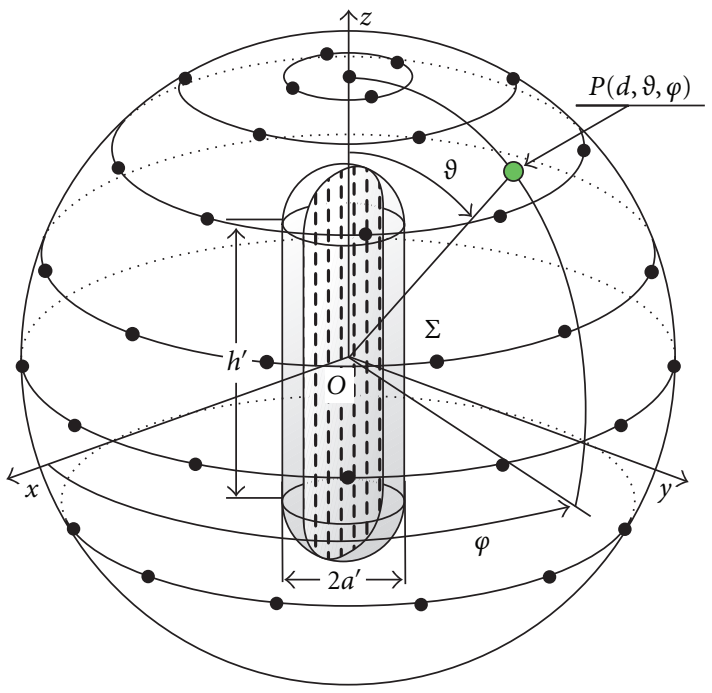

FIGURE 3: Spherical scanning for an elongated antenna.

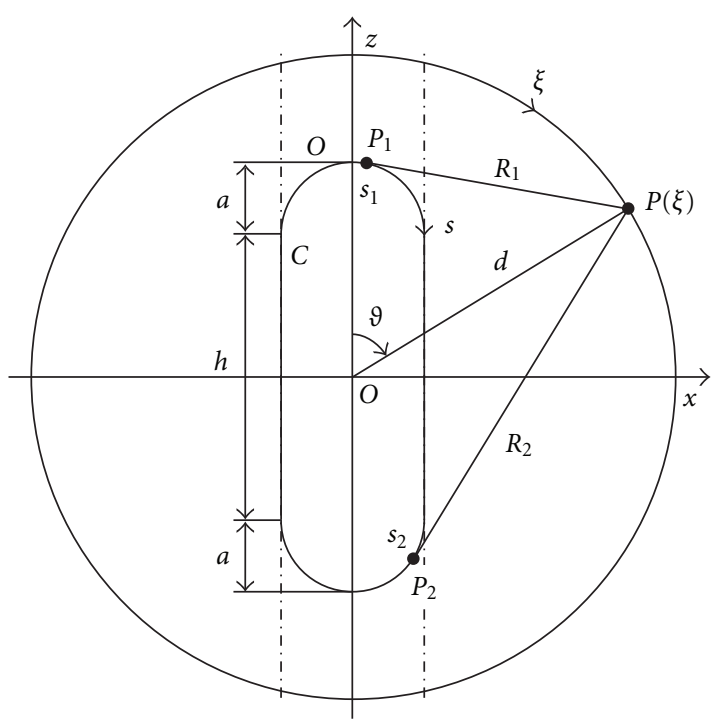

Figure 4: Relevant to the rounded cylinder modelling.

2.2. Rounded Cylinder Modelling. An effective modelling for an elongated AUT is got by considering it as enclosed in a cylinder of height $h^{\prime}$ ended in two half spheres of radius $a^{\prime}$ (see Figures 3 and 4). In such a case, it results that $\ell^{\prime}=$ $2\left(h^{\prime}+\pi a^{\prime}\right)$. The phase function and the optimal parameter relevant to a meridian can be again obtained by substituting the appropriate values of $R_{1,2}$ and $s_{1,2}^{\prime}$ in relations (3). It can be easily verified that their expressions change depending on the location of the tangency points $P_{1,2}$. In particular, three cases must be considered for $\vartheta$ ranging in $[0, \pi]$ (see Figure 4). 
When $0 \leq 9 \leq \sin ^{-1}\left(a^{\prime} / d\right)$, it results that

$$
\begin{gathered}
R_{1}=\sqrt{(d \sin \vartheta)^{2}+\left(d \cos \vartheta-\frac{h^{\prime}}{2}\right)^{2}-a^{\prime 2}}, \\
s_{1}^{\prime}=a^{\prime} \sin ^{-1}\left(\frac{a^{\prime} d \sin \vartheta+R_{1}\left(h^{\prime} / 2-d \cos \vartheta\right)}{R_{1}^{2}+a^{\prime 2}}\right), \\
R_{2}=R_{1}, \quad s_{2}^{\prime}=a^{\prime} \sin ^{-1}\left(\frac{a^{\prime} d \sin \vartheta-R_{2}\left(h^{\prime} / 2-d \cos \vartheta\right)}{R_{2}^{2}+a^{\prime 2}}\right) .
\end{gathered}
$$

When $\sin ^{-1}\left(a^{\prime} / d\right)<9 \leq \pi-\sin ^{-1}\left(a^{\prime} / d\right)$, the expressions of $R_{1}$ and $s_{1}^{\prime}$ are again given by relations (16), whereas it results that

$$
\begin{gathered}
R_{2}=\sqrt{(d \sin \vartheta)^{2}+\left(d \cos \vartheta+\frac{h^{\prime}}{2}\right)^{2}-a^{\prime 2}}, \\
s_{2}^{\prime}=h^{\prime}+a^{\prime}\left[\pi-\sin ^{-1}\left(\frac{a^{\prime} d \sin \vartheta+R_{2}\left(h^{\prime} / 2+d \cos \vartheta\right)}{R_{2}^{2}+a^{\prime 2}}\right)\right] .
\end{gathered}
$$

When $\pi-\sin ^{-1}\left(a^{\prime} / d\right)<\vartheta \leq \pi$, the expressions of $R_{2}$ and $s_{2}^{\prime}$ are again given by relations (17), whereas it results that

$$
\begin{gathered}
R_{1}=\sqrt{(d \sin \vartheta)^{2}+\left(d \cos \vartheta+\frac{h^{\prime}}{2}\right)^{2}-a^{\prime 2},} \\
s_{1}^{\prime}=h^{\prime}+a^{\prime}\left[\frac{\pi}{2}-\sin ^{-1}\left(\frac{R_{1} d \sin \vartheta+a^{\prime}\left(h^{\prime} / 2+d \cos \vartheta\right)}{R_{1}^{2}+a^{\prime 2}}\right)\right] .
\end{gathered}
$$

As regards the azimuthal bandwidth $W_{\varphi}$, it can be shown that the maximum in (4) is attained at

$$
z^{\prime}= \begin{cases}z, & |z| \leq \frac{h^{\prime}}{2}, \\ {\left[\frac{h^{\prime}}{2}+\frac{\left(|z|-h^{\prime} / 2\right) a^{\prime 2}}{(d \sin \vartheta)^{2}+\left(|z|-h^{\prime} / 2\right)^{2}}\right] \operatorname{sgn}(z),} & |z|>\frac{h^{\prime}}{2},\end{cases}
$$

where $\operatorname{sgn}(\cdot)$ is the sign function.

\section{NF-FF Transformation with Spherical Scanning}

For reader's convenience, the key steps of the probecompensated NF-FF transformation with spherical scanning [7] as modified in [15] by properly taking into account the spatial band-limitation properties of the EM field [16] are reported in this section.
According to [7], the electric field in the FF region can be expressed in terms of the following truncated spherical wave expansion:

$$
\begin{aligned}
\underline{E}(R, \Theta, \Phi)= & \frac{\mathrm{e}^{-j \beta R}}{R} \sum_{n=1}^{N_{\max }} \\
& \times \sum_{m=-n}^{n}\left[j^{n+1} b_{n m}^{1} \underline{f}_{1 n m}(\Theta)+j^{n} b_{n m}^{2} \underline{\tilde{f}}_{2 n m}(\Theta)\right] \\
& \times \mathrm{e}^{j m \Phi},
\end{aligned}
$$

where the spherical coordinate system $(R, \Theta, \Phi)$ has been adopted to specify the FF observation point.

The choice of the highest spherical wave to be considered is determined in the classical approach according to the following rule of thumb:

$$
N_{\max }=\operatorname{Int}(\beta a)+10,
$$

where $a$ is the radius of the smallest sphere enclosing the AUT. Whereas in the approach [15], the highest spherical harmonic is rigorously fixed by the aforementioned bandlimitation properties of the radiated EM fields [16]. Accordingly,

$$
N_{\max }=\operatorname{Int}\left(\chi^{\prime} \beta a\right)+1
$$

The vectorial functions $\underline{\tilde{f}}_{1,2 \mathrm{~nm}}(\Theta)$ are given by

$$
\begin{aligned}
& \underline{\tilde{f}}_{1 n m}(\Theta) \\
& =\left(-\frac{m}{|m|}\right)^{m} \frac{1}{\sqrt{2 \pi n(n+1)}} \\
& \quad \times\left[\frac{j m}{\sin \Theta} \bar{P}_{n}^{|m|}(\cos \Theta) \hat{\Theta}-\frac{d}{d \Theta} \bar{P}_{n}^{|m|}(\cos \Theta) \hat{\Phi}\right], \\
& \underline{\tilde{f}}_{2 n m}(\Theta) \\
& =\left(-\frac{m}{|m|}\right)^{m} \frac{1}{\sqrt{2 \pi n(n+1)}} \\
& \quad \times\left[\frac{\mathrm{d}}{\mathrm{d} \Theta} \bar{P}_{n}^{|m|}(\cos \Theta) \hat{\Theta}+\frac{j m}{\sin \Theta} \bar{P}_{n}^{|m|}(\cos \Theta) \hat{\Phi}\right],
\end{aligned}
$$

$\bar{P}_{n}^{|m|}(\cos \Theta)$ being the normalized associated Legendre functions as defined in [21].

As shown in [7], when using a probe with a first-order azimuthal dependence pattern (e.g., an open ended circular waveguide), the modal expansion coefficients in (20) can be determined from the knowledge of the voltages $V$ and $V^{\prime}$ 
measured by the probe and rotated probe, respectively, and are given by

$$
\begin{aligned}
& b_{n m}^{1}=\frac{2 n+1}{16 \pi} \frac{\int_{0}^{\pi} \int_{0}^{2 \pi}\left[I_{1}(\vartheta) V-j I_{2}(\vartheta) V^{\prime}\right] \mathrm{e}^{-j m \varphi} \sin \vartheta \mathrm{d} \varphi \mathrm{d} \vartheta}{\sum_{v=1}^{v_{\max }}\left[{b^{\prime}}^{\prime}{ }_{v 1} A_{v 1}^{n}(\beta d)-{b^{\prime}}^{\prime 2} B_{v 1}^{n}(\beta d)\right]}, \\
& b_{n m}^{2}=\frac{2 n+1}{16 \pi} \frac{\int_{0}^{\pi} \int_{0}^{2 \pi}\left[I_{2}(\vartheta) V-j I_{1}(\vartheta) V^{\prime}\right] \mathrm{e}^{-j m \varphi} \sin \vartheta d \varphi d \vartheta}{\sum_{v=1}^{v_{\max }}\left[{b^{\prime}}^{\prime 1} B_{v 1}^{n}(\beta d)-{b^{\prime}}^{\prime 2} A_{v 1}^{n}(\beta d)\right]},
\end{aligned}
$$

where $v_{\max }$ is the highest spherical wave of the probe,

$$
\begin{aligned}
& I_{1}(\vartheta)= {\left[d_{1 m}^{n}(\vartheta)-d_{-1 m}^{n}(\vartheta)\right], } \\
& I_{2}(\vartheta)=\left[d_{1 m}^{n}(\vartheta)+d_{-1 m}^{n}(\vartheta)\right], \\
& d_{\mu m}^{n}(\vartheta)= \sqrt{\frac{(n+\mu) !(n-\mu) !}{(n+m) !(n-m) !} \sum_{\sigma}\left(\begin{array}{c}
n+m \\
n-\mu-\sigma
\end{array}\right)} \\
& \times\left(\begin{array}{c}
n-m \\
\sigma
\end{array}\right)(-1)^{n-\mu-\sigma} \\
& \times\left(\cos \frac{\vartheta}{2}\right)^{2 \sigma+\mu+m}\left(\sin \frac{\vartheta}{2}\right)^{2 n-2 \sigma-\mu-m}
\end{aligned}
$$

are the rotation coefficients $[7,22]$, the summation over $\sigma$ involving all terms in which the binomial coefficients do not lead to negative arguments for the factorials. Moreover,

$$
\begin{aligned}
A_{v \mu}^{n}(\beta d)= & \sqrt{\frac{(2 n+1)(2 v+1)}{n(n+1) v(v+1)}} \sqrt{\frac{(v+\mu) !(n-\mu) !}{(v-\mu) !(n+\mu) !}} \\
& \times(-1)^{\mu} \frac{1}{2} j^{n-v} \sum_{p=|n-v|}^{n+v} \\
& \times\left\{j^{-p[n(n+1)+v(v+1)-p(p+1)]}\right. \\
B_{v \mu}^{n}(\beta d)= & \sqrt{\frac{(2 n+1)(2 v+1)}{n(n+1) v(v+1)} \sqrt{\frac{(v+\mu) !(n-\mu) !}{(v-\mu) !(n+\mu) !}}} \\
& \times(-1)^{\mu} \frac{1}{2} j^{n-v} \sum_{p=|n-v|}^{n+v} \\
& \times\left[j^{-p}(2 j \mu \beta d) \tau(\mu, n,-\mu, v, p) h_{p}^{(2)}(\beta d)\right]
\end{aligned}
$$

are the translation coefficients $[7,11,23]$, wherein $h_{p}^{(2)}(x)$ is the spherical Hankel function of second kind, and $\tau(\mu, n,-\mu, v, p)$ are the linearization coefficients defined by the expansion of the product of two unnormalized associated Legendre functions

$$
P_{n}^{\mu}(x) P_{v}^{-\mu}(x)=\sum_{p=|n-v|}^{n+v} \tau(\mu, n,-\mu, v, p) P_{p}(x) .
$$

The probe's expansion coefficients ${b^{\prime}}_{v 1}^{\prime, 2}$ in relations (24) can be evaluated from the knowledge of its tangential electric field on a scanning sphere when it is used as transmitting antenna

$$
\begin{aligned}
b_{v 1}^{\prime 1,2}= & \frac{1}{\beta g_{1,2 v}(\beta d)} \int_{0}^{\pi} \int_{-\pi}^{\pi} \underline{E}_{\mathrm{t}}^{\prime}(\mathrm{d}, \vartheta, \varphi) \cdot \underline{\tilde{f}}_{1,2 v 1}^{*}(\vartheta) \\
& \times \mathrm{e}^{-j \varphi} \sin \vartheta \mathrm{d} \varphi \mathrm{d} \vartheta
\end{aligned}
$$

$(\cdot)$ and $(*)$ denoting the inner product and the complex conjugate, respectively, and

$$
g_{1, v}(x)=h_{v}^{(2)}(x), \quad g_{2, v}(x)=\frac{1}{x} \frac{d}{\mathrm{~d} x}\left[x h_{v}^{(2)}(x)\right] .
$$

According to (28), the probe's expansion coefficients can be determined from the simulated measurements of the field radiated by an open-ended circular waveguide by using the uncompensated NF-FF transformation [14].

The integration over $\varphi$ in relations (24) can be carried out [14] by expanding the voltage measured by the probe and rotated probe on each parallel in Fourier series with respect to $\varphi$, and this can be efficiently performed by employing the Fast Fourier Transform (FFT) algorithm. In a quite similar way, the remaining integration over $\vartheta$ can be efficiently performed through the following steps: (i) the aforementioned Fourier series coefficients are extended [7] from the range $[0, \pi]$ to $[0,2 \pi]$ and then expanded in Fourier series via FFT, (ii) the rotation coefficients are expanded in Fourier series too [7, 22]. The double integrals are so transformed in double summations, of indexes $\ell$ and $i$, involving the Fourier series coefficients (in $\vartheta$ ) and the integrals

$$
\int_{0}^{\pi} \mathrm{e}^{j(\ell-i) \vartheta} \sin \vartheta \mathrm{d} \vartheta
$$

It must be stressed that, to take advantage of the numerical efficiency of the FFT, the number of NF parallels to be considered in the NF-FF transformation and the number of samples on them must be the first power of two greater or equal to $N_{\max }$ and $2 M_{\varphi}$, respectively, where $M_{\varphi}=$ $\operatorname{Int}\left(\chi^{*} \beta a \sin \vartheta\right)+1$.

It is worth noting that, by inverting the summations order, the spherical wave expansion (20) can be rewritten as

$$
\begin{aligned}
\underline{E}(R, \Theta, \Phi)= & \frac{\mathrm{e}^{-j \beta R}}{R} \sum_{m=-M_{\Phi}}^{M_{\Phi}} \sum_{\substack{n=|m| \\
(n \neq 0)}}^{N_{\max }} \\
& \times\left[j^{n+1} b_{n m}^{1} \underline{\tilde{f}}_{1 n m}(\Theta)+j^{n} b_{n m}^{2} \underline{\tilde{f}}_{2 n m}(\Theta)\right] \mathrm{e}^{j m \Phi} \\
= & \frac{\mathrm{e}^{-j \beta R}}{R} \sum_{m=-M_{\Phi}}^{M_{\Phi}} \underline{c}_{m}(\Theta) \mathrm{e}^{j m \Phi},
\end{aligned}
$$

where $M_{\Phi}=\operatorname{Int}\left(\chi^{*} \beta a \sin \Theta\right)+1$. Relation (31) allows an efficient evaluation of the antenna far field at the considered elevation angle $\Theta$ by carrying out the summation again via the FFT algorithm. 
TABLE 1

\begin{tabular}{lc}
\hline NF-FF transformation technique & $N_{\text {data }}$ \\
\hline $\begin{array}{l}\text { Standard approach [7] (same number of data on each } \\
\text { parallel) }\end{array}$ & 130562 \\
$\begin{array}{l}\text { Standard approach as modified in [15] (number of data } \\
\text { on rings decreasing towards the poles) }\end{array}$ & 80610 \\
Here proposed approach & 26467 \\
\hline
\end{tabular}

\section{TABLE 2}

\begin{tabular}{lc}
\hline NF-FF transformation technique & $N_{\text {data }}$ \\
\hline $\begin{array}{l}\text { Standard approach [7] (same number of data on each } \\
\text { parallel) }\end{array}$ & 130562 \\
$\begin{array}{l}\text { Standard approach as modified in [15] (number of data } \\
\text { on rings decreasing towards the poles) }\end{array}$ & 102786 \\
\begin{tabular}{l} 
Here proposed approach \\
\hline
\end{tabular} & 27231 \\
\hline
\end{tabular}

From a numerical viewpoint, it is convenient to apply the described FF reconstruction process to evaluate only the FF samples required by the OSI expansion in [24], properly modified to deal with an even number of samples on the FF parallels:

$\underline{F}(\Theta, \Phi)$

$$
\begin{aligned}
=\sum_{m=m_{0}-q+1}^{m_{0}+q}\left\{\Omega_{N}\left(\Theta-\Theta_{m}\right) D_{N^{\prime \prime}}\left(\Theta-\Theta_{m}\right)\right. \\
\quad \times \frac{2 L_{\Phi}^{\prime \prime}-1}{2 L_{\Phi}^{\prime \prime}} \sum_{n=n_{0}-p+1}^{n_{0}+p} \underline{F}\left(\Theta_{m}, \Phi_{n, m}\right) \\
\left.\quad \times \Omega_{L_{\Phi}}\left(\Phi-\Phi_{n, m}\right) D_{L_{\Phi}^{\prime \prime}-1}\left(\Phi-\Phi_{n, m}\right)\right\}
\end{aligned}
$$

wherein

$$
\begin{gathered}
\Theta_{m}=m \Delta \Theta=\frac{2 \pi m}{\left(2 N^{\prime \prime}+1\right)}, \\
N^{\prime \prime}=\operatorname{Int}\left(\chi N_{\max }\right)+1, \quad N=N^{\prime \prime}-N_{\max }, \\
\Phi_{n, m}=n \Delta \Phi_{m}=n \frac{\pi}{L_{\Phi}^{\prime \prime}}, \\
L_{\Phi}^{\prime \prime}=2^{i} \geq \operatorname{Int}\left(\chi M_{\Phi}\right)+1, \quad L_{\Phi}=L_{\Phi}^{\prime \prime}-M_{\Phi},
\end{gathered}
$$

$\underline{F}(\Theta, \Phi)=R \mathrm{e}^{\mathrm{j} \beta R} \underline{E}(R, \Theta, \Phi)$, and the other symbols have the same or analogous meanings as in (10).

It is useful to note that the expansion (32) is based on a spherical modelling of the antenna. OSI expansions requiring a lower number of FF samples can be obviously derived when using more effective modellings tailored to the actual antenna geometry.

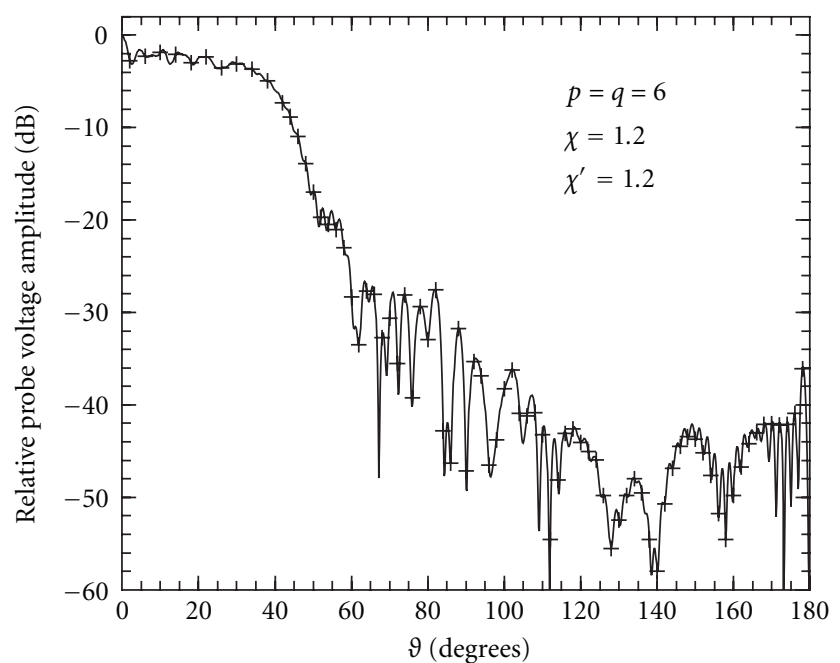

Figure 5: Amplitude of the output voltage $V^{\prime}$ on the meridian at $\varphi=90^{\circ}$. Solid line: exact. Crosses: interpolated.

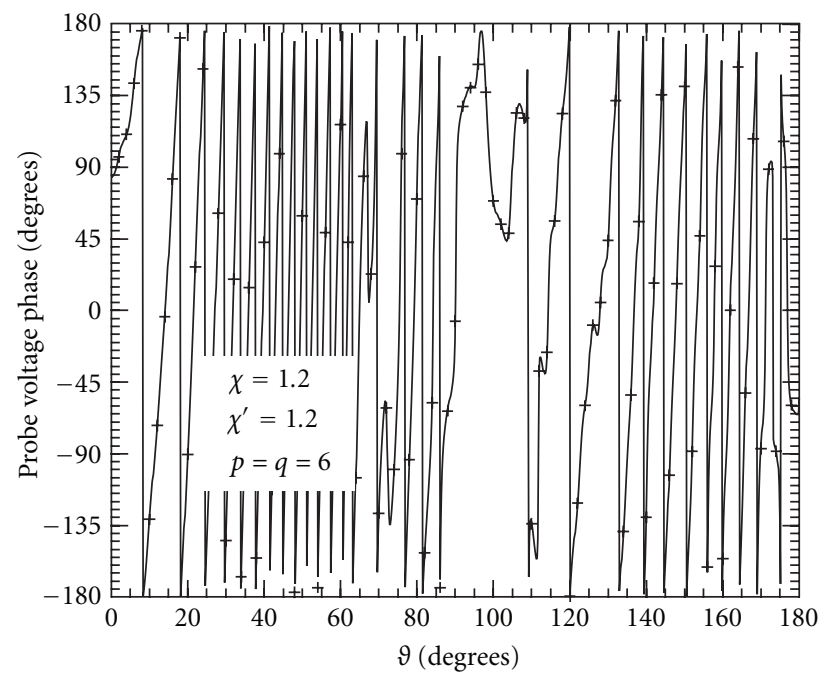

Figure 6: Phase of the output voltage $V^{\prime}$ on the meridian at $\varphi=$ $90^{\circ}$. Solid line: exact. Crosses: interpolated.

\section{Numerical Tests}

Many numerical simulations have been performed in order to assess the effectiveness and robustness of the proposed NF-FF transformation techniques with spherical scanning for antennas having two dimensions very different from the third one. Two sets of simulations are reported in the following. The former (from Figure 5 to 11) refers to a quasiplanar antenna, whereas the latter (from Figure 12 to 16) is relevant to the case of an elongated one. In both the cases, an open-ended circular waveguide having radius $0.338 \lambda, \lambda$ being the wavelength is chosen as measurement probe.

In the first set, the numerical tests refer to a scanning sphere having radius $d=25 \lambda$ and to three uniform planar circular arrays placed at $z=-2 \lambda, 1 \lambda, 4 \lambda$, having radius 


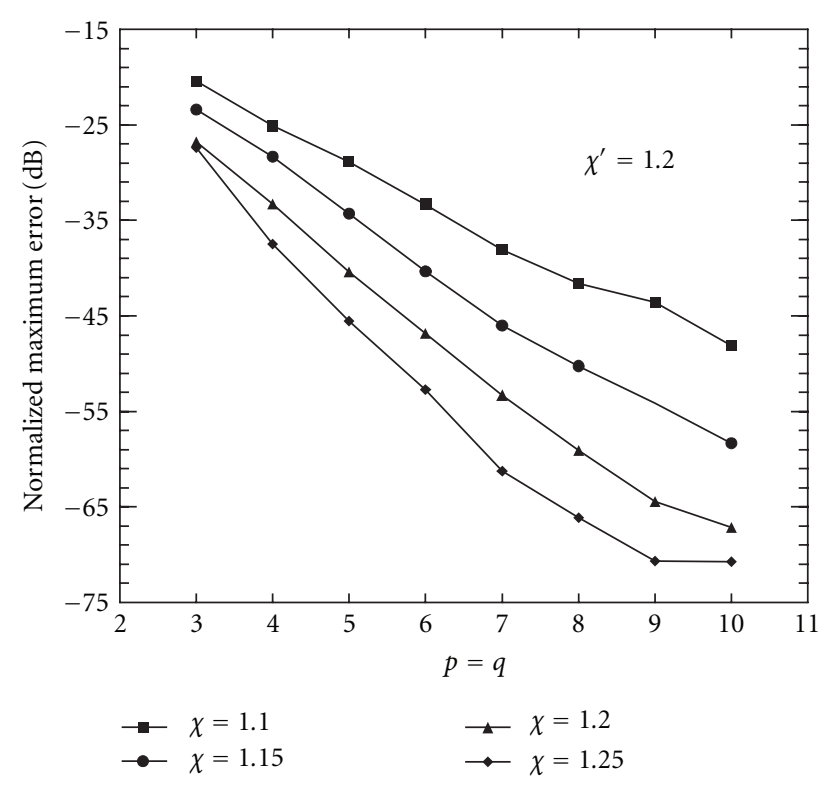

Figure 7: Normalized maximum errors in the reconstruction of the voltage $V^{\prime}$.

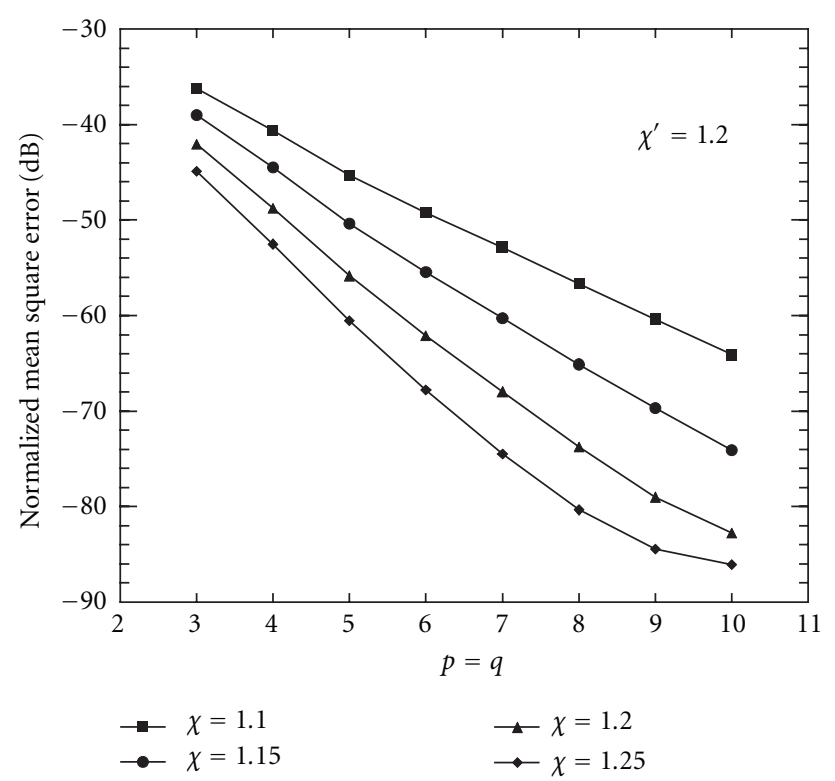

FIGURE 8: Normalized mean-square errors in the reconstruction of the voltage $V^{\prime}$.

equal to $18 \lambda, 19.5 \lambda$, and $16 \lambda$, respectively. Their elements, symmetrically placed with respect to the plane $y=0$, are elementary Huygens sources linearly polarized along $y$ and spaced of $0.5 \lambda$ along the radial and azimuthal lines. Such an AUT has been fitted by the double bowl modelling with $a=20 \lambda, c=4.5 \lambda$, and $c^{\prime}=2.5 \lambda$. Figures 5 and 6 show representative reconstruction examples of the amplitude and phase of the rotated probe voltage $V^{\prime}$ on the meridian at $\varphi=90^{\circ}$. As can be seen, the exact and recovered voltages are practically indistinguishable. To assess in a more quantitative

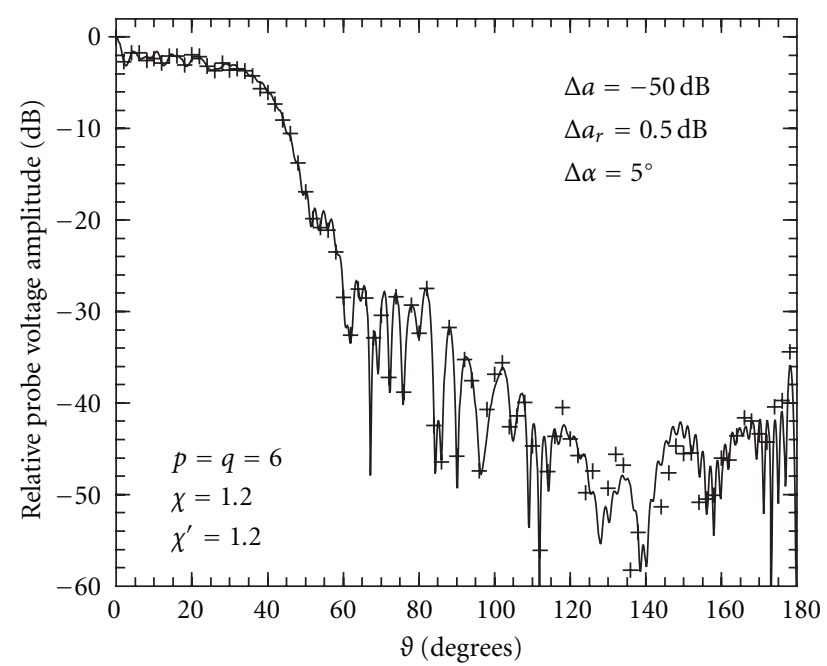

FIGURE 9: Amplitude of the output voltage $V^{\prime}$ on the meridian at $\varphi=90^{\circ}$. Solid line: exact. Crosses: interpolated from error-affected data.

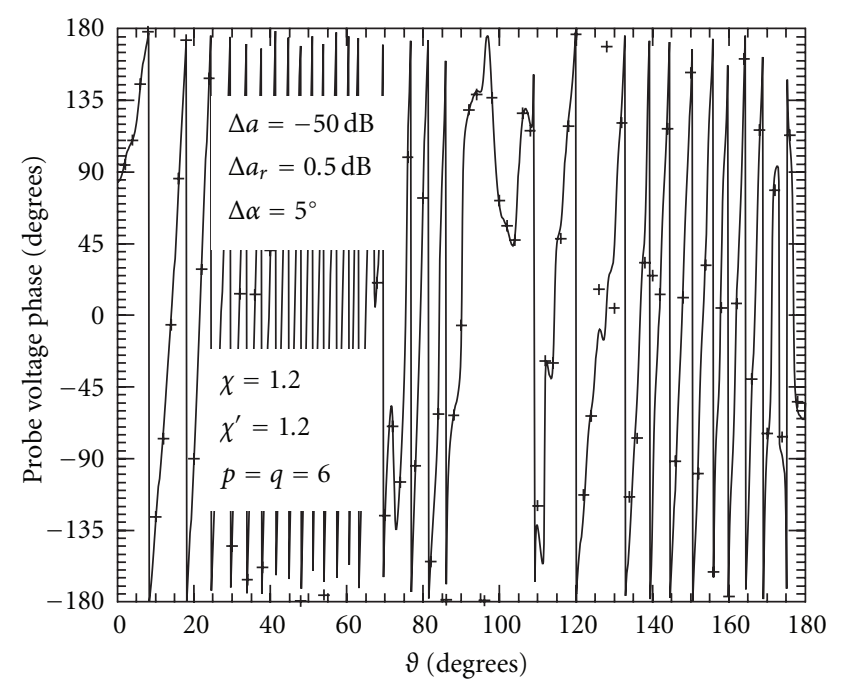

Figure 10: Phase of the output voltage $V^{\prime}$ on the meridian at $\varphi=$ $90^{\circ}$. Solid line: exact. Crosses: interpolated from error-affected data.

way the performances of the algorithm, the maximum and mean-square reconstruction errors (normalized to the voltage maximum value over the sphere) have been evaluated for $\chi^{\prime}=1.20, \chi=1.10,1.15,1.20,1.25$, and $p=q$ ranging from 3 to 10 (see Figures. 7 and 8). They have been computed by comparing the reconstructed and exact voltage $V^{\prime}$ on a spherical grid with $\Delta \vartheta=\Delta \varphi=1^{\circ}$. As can be seen, the errors decrease up to very low values on increasing the oversampling factor and/or the number of retained samples, thus assessing the effectiveness of the interpolation procedure. The algorithm robustness has been verified by adding random errors to the exact samples. These errors simulate a background noise (bounded to $\Delta a$ in amplitude and with arbitrary phase) and an uncertainty on the voltage samples of $\pm \Delta a_{r}$ in amplitude and $\pm \Delta \alpha$ in 


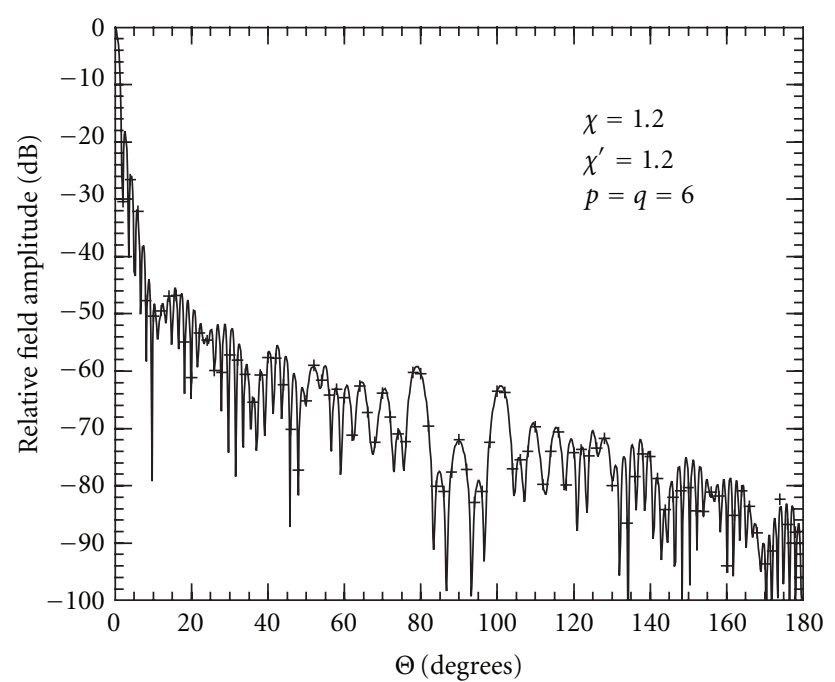

FIGURE 11: E-plane pattern. Solid line: exact. Crosses: reconstructed from NF data.

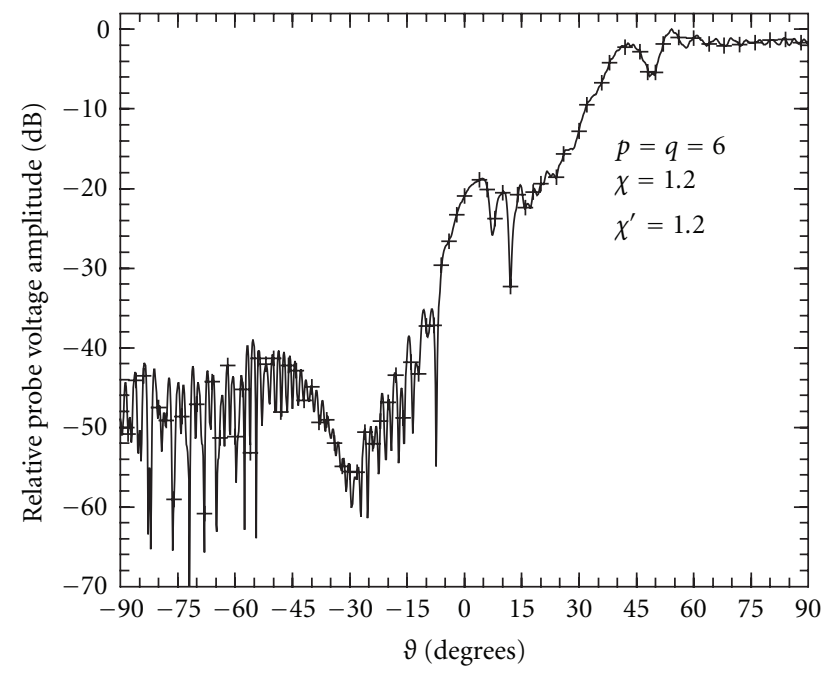

Figure 12: Amplitude of the output voltage $V^{\prime}$ on the meridian at $\varphi=90^{\circ}$. Solid line: exact. Crosses: interpolated.

phase. As shown in Figures 9 and 10, the algorithm works well also when dealing with error-affected data. At last, the developed interpolation algorithm has been applied to recover the NF data required to carry out the spherical NF-FF transformation [7], as modified in [15]. The reconstruction of the antenna FF pattern in the principal plane $\mathrm{E}$ is shown in Figure 11. As can be seen, the FF reconstruction, in spite of the significantly reduced number of used NF samples (see Table 1), is everywhere very accurate thus validating the effectiveness of the proposed approach.

The second set of figures refers to a scanning sphere having radius $d=35 \lambda$ and to a uniform planar array of elementary Huygens sources polarized along the $z$ axis, spaced by $0.5 \lambda$. These sources cover a zone in the plane $y=0$ formed by a $16 \lambda \times 44 \lambda$ rectangle ended in two halfcircles. Accordingly, such an antenna has been modelled

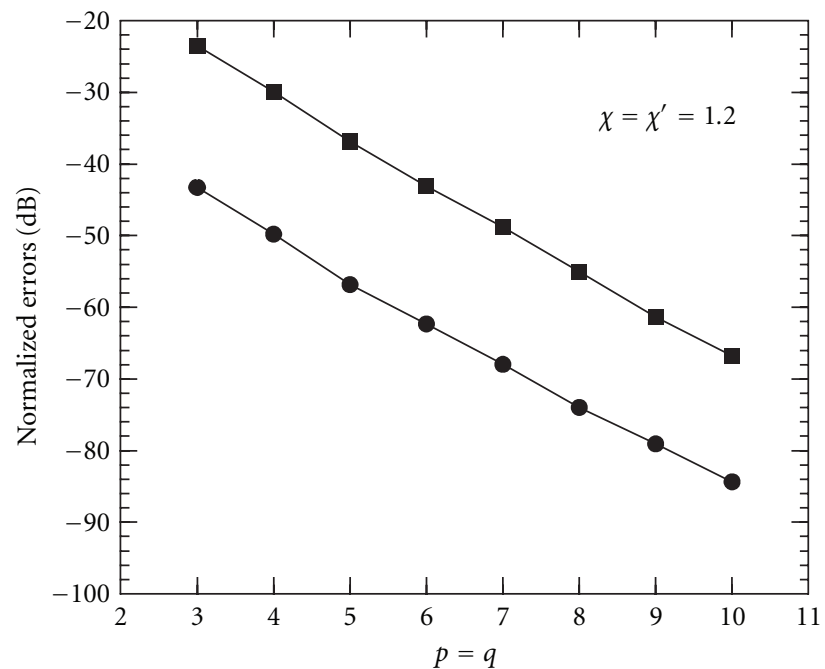

Maximum error

Mean-square error

FIGURE 13: Normalized errors in the reconstruction of the voltage $V^{\prime}$.

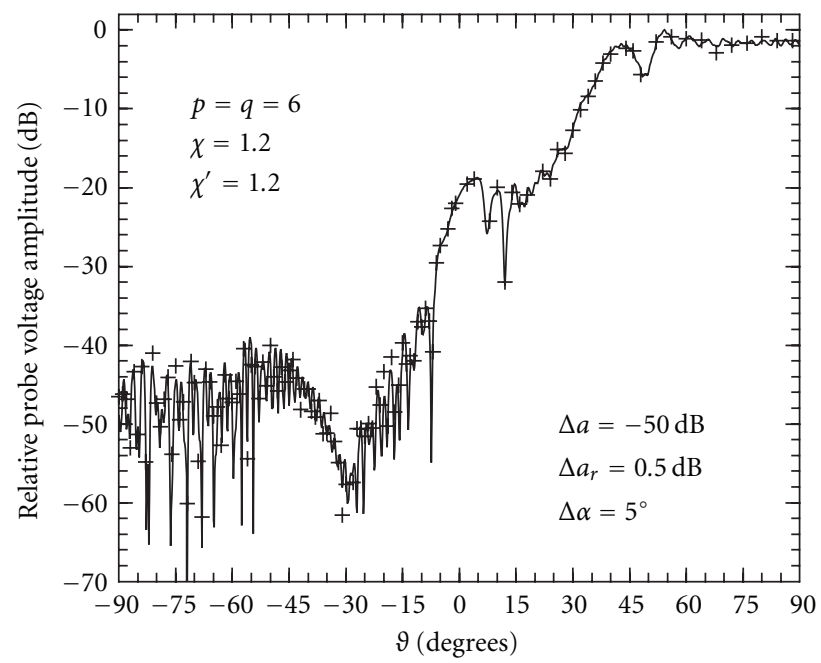

Figure 14: Amplitude of the output voltage $V^{\prime}$ on the meridian at $\varphi=90^{\circ}$. Solid line: exact. Crosses: interpolated from error-affected data.

by a rounded cylinder with $h^{\prime}=44 \lambda$ and $a^{\prime}=8 \lambda$. An example of voltage reconstruction relevant to the meridian at $\varphi=90^{\circ}$ is reported in Figure 12. Again, a very accurate reconstruction results. Figure 13 confirms the algorithm accuracy by showing the normalized maximum and meansquare errors for $p=q$ ranging from 3 to 10 and $\chi^{\prime}=$ $\chi=1.20$. Also in such a case, the algorithm is resulted to be very stable with respect to random errors affecting the exact samples as shown in Figure 14. At last, the reconstructions of the antenna FF pattern in the $\mathrm{E}$ and $\mathrm{H}$ planes are shown in Figures 15 and 16, respectively. As can be seen, the exact and recovered fields are practically indistinguishable, thus assessing the effectiveness of the developed NF-FF 


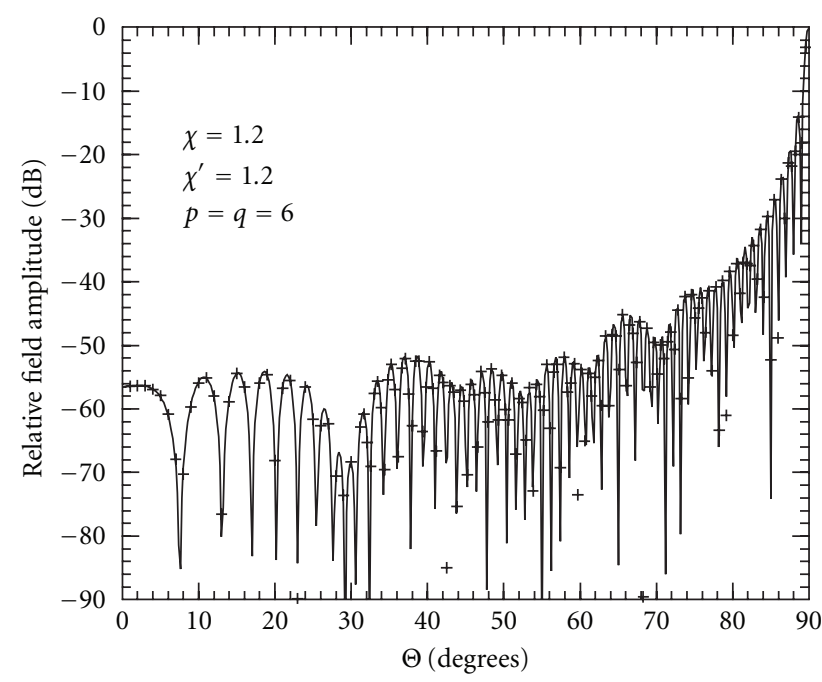

FIGURE 15: E-plane pattern. Solid line: exact. Crosses: reconstructed from NF data.

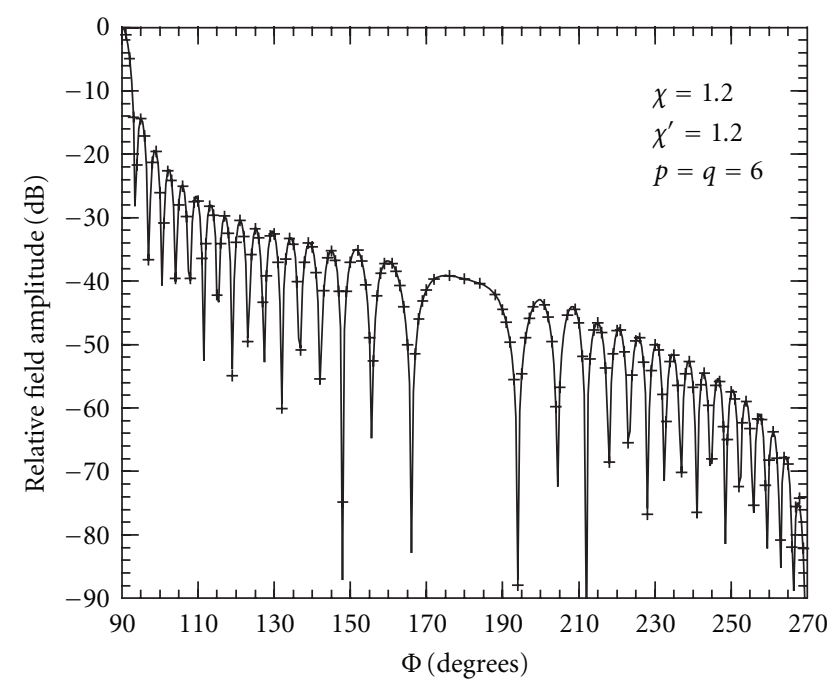

Figure 16: H-plane pattern. Solid line: exact. Crosses: reconstructed from NF data.

transformation. Also in such a case, it can be interesting to compare the number of the employed NF data with those required by the other techniques (see Table 2 ).

\section{Conclusions}

Fast and accurate probe-compensated NF-FF transformations with spherical scanning for nonspherical antennas have been here developed by employing flexible AUT modellings, which allow one to fit very well the shape of a lot of actual antennas. In particular, an electrically long antenna has been considered as enclosed in a rounded cylinder, whereas a double bowl has been adopted to shape a quasiplanar antenna. The use of these modellings makes possible to remarkably lower the number of data to be acquired, thus significantly reducing the measurement time.
The developed techniques work very well as widely assessed by the numerical simulations.

\section{References}

[1] A. D. Yaghjian, "An overview of near-field antenna measurements," IEEE Transactions on Antennas and Propagation, vol. 34 , no. 1, pp. 30-45, 1986.

[2] "Special Issue on near-field scanning techniques," IEEE Transactions on Antennas and Propagation, vol. 36, no. 6, pp. 727901, 1988.

[3] C. Gennarelli, G. Riccio, F. D'Agostino, and F. Ferrara, NearField-Far-Field Transformation Techniques, vol. 1, CUES, Salerno, Italy, 2004.

[4] C. A. Balanis, Modern Antenna Handbook, John Wiley \& Sons, Hoboken, NJ, USA, 2008.

[5] P. Petre and T. K. Sarkar, "Differences between modal expansion and integral equation methods for planar nearfield to far-field transformation," Progress in Electromagnetics Research, vol. 12, pp. 37-56, 1996.

[6] Y. Álvarez, F. Las-Heras, and M. R. Pino, "Probe-distortion correction for the sources reconstruction method," IEEE Antennas and Propagation Magazine, vol. 50, no. 6, pp. 117124, 2008.

[7] J. Hald, J. E. Hansen, F. Jensen, and F. H. Larsen, Spherical Near-Field Antenna Measurements, J. E. Hansen Ed., IEE Electromagnetic Waves Series, Peter Peregrinus, London, UK, 1998.

[8] F. Jensen, Electromagnetic near-field-far-field correlations, Ph.D. dissertation, Technical University of Denmark, 1970.

[9] P. F. Wacker, "Non-planar near-field measurements: spherical scanning," Tech. Rep. NBSIR 75-809, Boulder, Colo, USA, 1975.

[10] F. H. Larsen, "Probe correction of spherical near-field measurements," Electronics Letters, vol. 13, no. 14, pp. 393-395, 1977.

[11] F. H. Larsen, Probe-corrected spherical near-field antenna measurements, Ph.D. dissertation, Technical University of Denmark, 1980.

[12] A. D. Yaghjian and R. C. Wittmann, "The receiving antenna as a linear differential operator: application to spherical near-field measurements," IEEE Transactions on Antennas and Propagation, vol. 33, no. 11, pp. 1175-1185, 1985.

[13] J. E. Hansen and F. Jensen, "Spherical near-field scanning at the Technical University of Denmark," IEEE Transactions on Antennas and Propagation, vol. 36, no. 6, pp. 734-739, 1988.

[14] O. M. Bucci, F. D’Agostino, C. Gennarelli, G. Riccio, and C. Savarese, "Data reduction in the NF-FF transformation technique with spherical scanning," Journal of Electromagnetic Waves and Applications, vol. 15, no. 6, pp. 755-775, 2001.

[15] A. Arena, F. D’Agostino, C. Gennarelli, and G. Riccio, "Probe compensated NF-FF transformation with spherical scanning from a minimum number of data," Atti della Fondazione G. Ronchi, vol. 59, no. 3, pp. 312-326, 2004.

[16] O. M. Bucci and G. Franceschetti, "On the spatial bandwidth of scattered fields," IEEE Transactions on Antennas and Propagation, vol. 35, no. 12, pp. 1445-1455, 1987.

[17] O. M. Bucci, C. Gennarelli, and C. Savarese, "Representation of electromagnetic fields over arbitrary surfaces by a finite and nonredundant number of samples," IEEE Transactions on Antennas and Propagation, vol. 46, no. 3, pp. 351-359, 1998.

[18] J. C. Bolomey, B. J. Cown, G. Fine et al., "Rapid near-field antenna testing via arrays of modulated scattering probes," 
IEEE Transactions on Antennas and Propagation, vol. 36, no. 6, pp. 804-814, 1988.

[19] O. M. Bucci, G. D’Elia, and M. D. Migliore, "Advanced field interpolation from plane-polar samples: experimental verification," IEEE Transactions on Antennas and Propagation, vol. 46, no. 2, pp. 204-210, 1998.

[20] C. Gennarelli, G. Riccio, V. Speranza, and C. Savarese, "Fast and accurate interpo-lation of radiated fields over a cylinder," Progress in Electromagnetics Research, vol. 8, pp. 349-375, 1994.

[21] S. L. Belousov, Tables of Normalized Associated Legendre Polynomials, Pergamon Press, Oxford, UK, 1962.

[22] A. R. Edmonds, Angular Momentum in Quantum Mechanics, Princeton University Press, Princeton, NJ, USA, 1974.

[23] J. H. Bruning and Y. T. Lo, "Multiple scattering of EM waves by spheres-part 1: multipole expansion and ray-optical solutions," IEEE Transactions on Antennas and Propagation, vol. 19, no. 3, pp. 378-390, 1971.

[24] O. M. Bucci, C. Gennarelli, and C. Savarese, "Optimal interpolation of radiated fields over a sphere," IEEE Transactions on Antennas and Propagation, vol. 39, no. 11, pp. 1633-1643, 1991. 

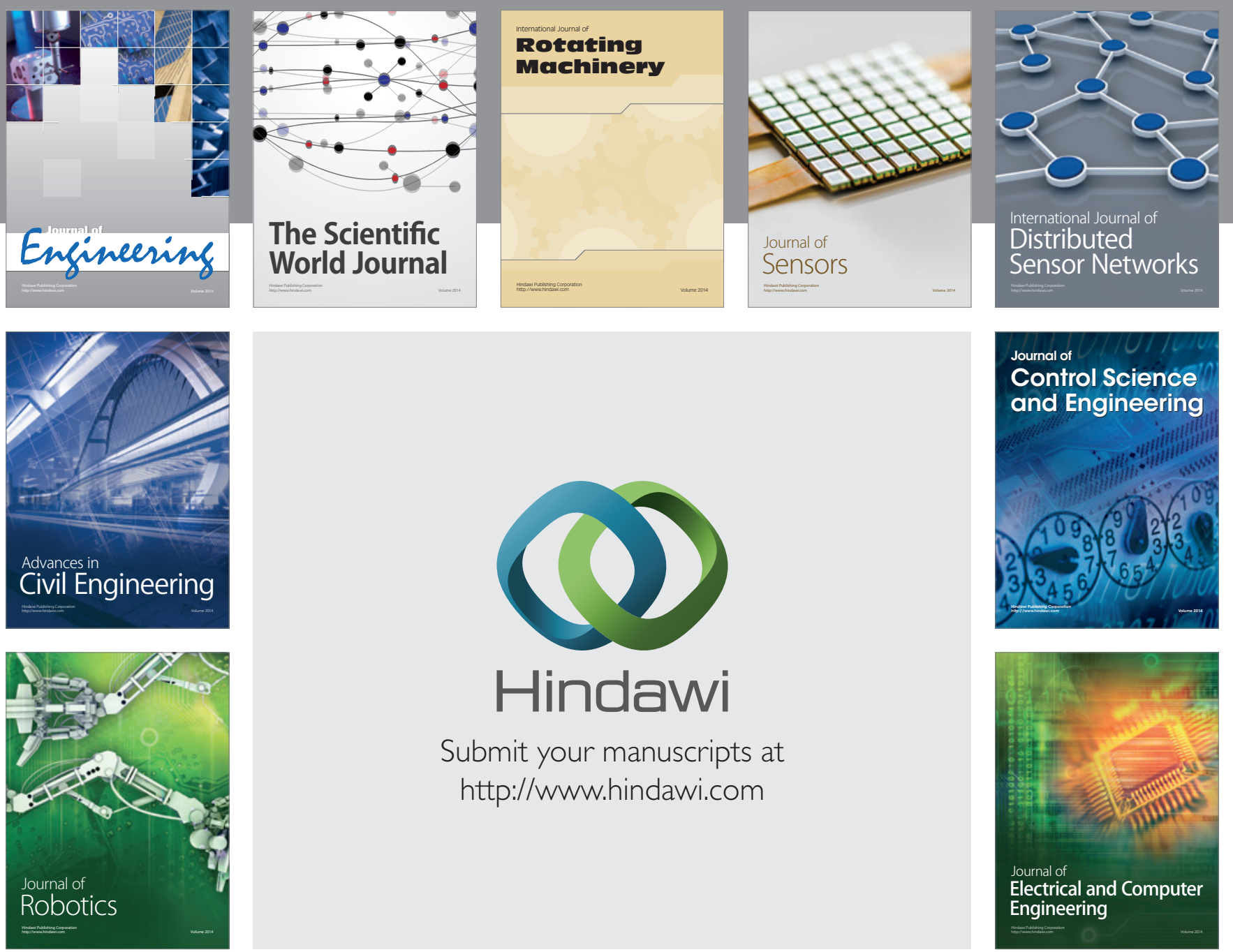

Submit your manuscripts at

http://www.hindawi.com
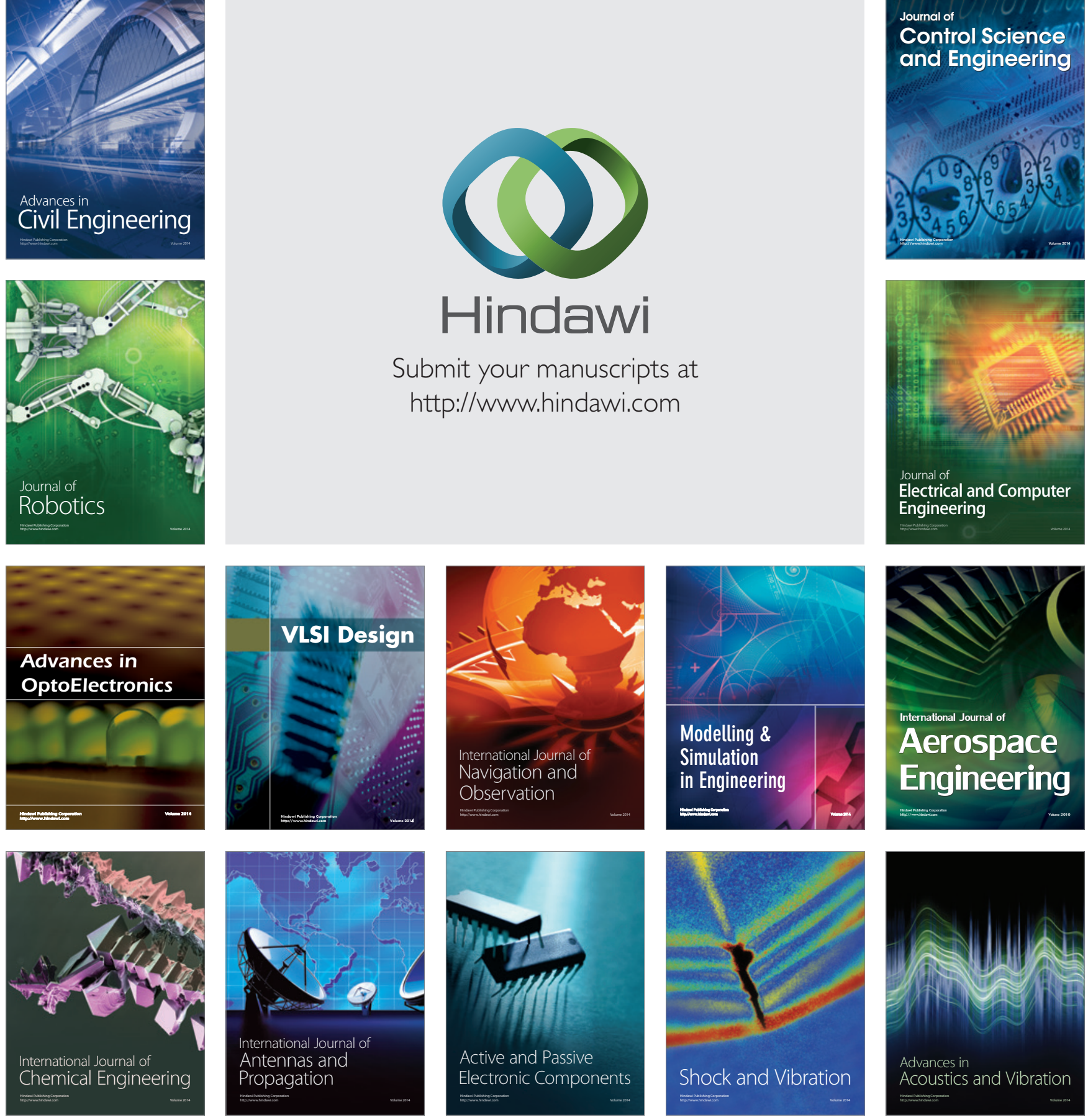